\title{
17. THE SOLUTION OF PROBLEMS OF COMETARY ASTRONOMY ON ELECTRONIC COMPUTERS
}

\author{
N. A. BELYAEV \\ Institute for Theoretical Astronomy, Leningrad, U.S.S.R.
}

\begin{abstract}
A series of standard programmes has been developed for numerical integration by Cowell's method of the differential equations of motion of minor bodies. A variable step is used, and perturbations by eight major planets and nongravitational effects are taken into consideration. Further programmes have been constructed as part of a general attempt at ITA to produce numerical theories of cometary motion. They include the reduction of observations, the comparison of the observations with theory and the improvement of orbits. The programmes make it possible to calculate $(\mathrm{O}-\mathrm{C})$ residuals of up to 2000 observations simultaneously.
\end{abstract}

The study of the motions of comets and other minor bodies of the solar system is one of the major problems investigated at the Institute for Theoretical Astronomy of the Academy of Sciences of the U.S.S.R. Accordingly, great attention is paid at the Institute to developing various methods of integration and constructing the sets of programmes needed for solving a wide range of problems in cometary and meteor astronomy. Each of these sets has its own method of integration and manner of allowing for perturbations (Herrick, Cowell, Numerov, Myachin, etc.). The programmes that have been developed supplement one another, and this helps to ensure that the results obtained are reliable to the accuracy required. The possibility of choosing a method according to the orbital characteristics, accuracy of the starting data and other factors leads to the greatest compatibility between the method of investigation and the objective.

Following Bokhan's (1972) initiative, in order to reduce the time needed for programming we have introduced a process of standardization of astronomical calculations and have compiled at the Institute a large library of standard programmes (SP's) on punched cards, this being intended for use in the solution of a wide variety of problems. This method of programming appreciably facilitated the construction of large programmes, for any complicated programme is now to some degree the result of mutual activity.

Our set of BESM-4 computer programmes devised for solving the problems of cometary astronomy includes programmes for processing observations, comparison of observations with orbits, improvement of elliptical orbits, computation of ephemerides, and study of the evolution of cometary orbits.

The numerical integration is done by Cowell's method, using a variable step and allowing for up to the fourth differences. The perturbations are computed using a series of osculating elements of the eight major planets Venus-Pluto. The frequency of the epochs of osculation was chosen separately for each planet and such that coordinates calculated from the formulae for undisturbed motion could give the required accuracy at any instant during the 400-year interval 1660 to 2060. The whole series of elements was thoroughly checked. 
For checking the integration step we apply Kulikov's (1964) criterion, namely, that the fourth difference $\bar{f}_{0}^{4}$ satisfies

$$
2^{p+3} \varepsilon_{0}<\left|\bar{f}_{0}^{4}\right|<2^{p+9} \varepsilon_{0},
$$

where $p$ gives the order of the first sum (in binary), and $\varepsilon_{0}$ is the permissible relative error of the calculations. If the lower limit of this inequality does not hold, the integration step is doubled, and if the upper limit does not hold, it is halved.

Table I shows the variation of the integration step for P/Giacobini-Zinner during 1933-1940. The total number of steps for one revolution is 194 , so that the 'mean' step is 12 days. A comet with the same period but a perihelion distance of $1.5 \mathrm{AU}$ or more would have a mean step of 15 days. The maximum step for minor bodies of the solar system is $\mathbf{4 0}$ days.

\section{TABLE I}

Variation with heliocentric distance $r$ of the integration steps $\tilde{\omega}$ for P/Giacobini-Zinner during the interval $1933-1940$

\begin{tabular}{lcl}
\hline$\tilde{\omega}$ (days) & $r$ (AU) & No. of steps \\
\hline 5 & 1.46 & 23 \\
10 & 2.50 & 22 \\
20 & 4.03 & 18 \\
40 & 5.48 & 25 \\
20 & $(6.03)$ & 16 \\
10 & 5.05 & 16 \\
5 & 3.44 & 20 \\
2.5 & 2.19 & 54 \\
5 & 1.27 & - \\
\hline
\end{tabular}

$e=0.6, q=1.0 \mathrm{AU}, Q=6.0 \mathrm{AU}$, $P \sim 2400$ days.

It is well known that when integrating with a variable step it is desirable to store in the computer's memory the precise coordinates of the body for up to 12 or 13 integration points. It is then possible to recalculate the coordinates without loss of accuracy at any point in the integration table. Our programme has been so constructed that the operative memory of the computer stores the precise coordinates for as few as seven integration points, about half the usual number. This was achieved by means of a new method for the recalculation of the integration table. The method 
involves a new table constructed from osculating elements that are obtained from the precise coordinates (for seven points) given in the previous table. The perturbations are allowed for by means of the formulae by Kazimirchak-Polonskaya (1967). These formulae are intended for calculating the planetary perturbations in rectangular coordinates for five initial integration points, provided that the epoch of osculation $T_{0}$ and integration step $\tilde{\omega}$ are taken to be arbitrary. The perturbations are extrapolated for the two extreme points. The calculation involves successive approximations until the differences between two consecutive iterations are less than the relative accuracy required.

If necessary, the nongravitational effects are taken into consideration by the formulae (Dubyago, 1949)

$$
\begin{aligned}
\varphi & =\varphi_{0}+\varphi^{\prime}\left(t-t_{0}\right)+\text { perturbations } \\
\mu & =\mu_{0}+2 \mu^{\prime}\left(t-t_{0}\right)+\text { perturbations } \\
M & =M_{0}+\mu_{0}\left(t-t_{0}\right)+\mu^{\prime}\left(t-t_{0}\right)^{2}+\text { perturbations, }
\end{aligned}
$$

and changing the step near perihelion (heliocentric distance less than $3 \mathrm{AU}$ ), as a rule from $\tilde{\omega}=10$ to $\tilde{\omega}=5$ and vice versa.

In view of the great scope of future studies in cometary and meteor astronomy particular thought was given to the form of the computer input and output. A special standard programme was constructed so that the input data could be in any of about 30 different styles, which means that there is practically no need for any preliminary processing of information taken from the literature. Another programme causes the following quantities to be printed out:

$T_{0}$ (civil date), $T_{0}$ (Julian date), $M_{0}, \omega, \Omega, i, \varphi, \mu, e, a, q=a(1-e), Q=a(1+e)$,

$P$ (revolution period), $\pi=\omega+\Omega, r$ (heliocentric distance), $v_{0}$ (true anomaly), $l_{0}=\pi+v_{0}, C_{0}$ (Jacobian constant), $\Sigma$ (number of steps), $\tilde{\omega}$ (step).

These values, required for additional analysis, plotting, etc., are printed out at perihelion passage, for a number of preselected dates, at encounters with major planets and, if desired, at each integration step. The information output at approaches of comets to the major planets is controlled by a special programme that analyses the results for each separate planet before printing out.

Three programmes intended for comparison with observations, ephemeris computation and investigation of the evolution of cometary orbits were constructed on the basis of the integration programme described above. About $90 \%$ of these large programmes consist of the same standard programmes.

The solution of problems of cometary astronomy on electronic computers inevitably poses the problem of an increase in accuracy at all stages of the computations and, above all, in the starting data. We have therefore embarked at ITA on a project of constructing numerical theories of cometary motion, consideration being given to the entire range of observational material. This involves collecting and processing a great number of observations obtained during the last 100 to 120 years. To facilitate this work a scheme was devised for extracting observations from primary sources and cataloguing them, and a special programme was constructed for processing observa- 
tions. A rubber stamp was made for printing the scheme on the back side of computer cards. Experience at ITA and other institutions has shown that writing down each observation on a separate computer card is very convenient when compiling large catalogues of cometary observations.

The processing programme converts the time of observation to Ephemeris Time, refers $\alpha_{0}$ and $\delta_{0}$ to the standard equinox 1950.0, calculates the precise rectangular coordinates of the Sun and correction for parallax. The coordinates of the Sun are computed from Newcomb's expansians using a standard programme by Podunova. To allow for parallax, a catalogue of 500 observatories has been compiled from all the references at our disposal. The correction for aberration is taken into account in calculating the $(\mathrm{O}-\mathrm{C})$ residuals. Up to 2000 observations of a comet may be processed and compared at a time.

A programme for improving elliptical orbits by use of Eckert-Brouwer coefficients has also been constructed, starting from standard programmes by Bokhan, Makover and Nikol'skaya. Up to 200 observations or normal places may be utilized for the improvement. If the accuracy proves to be inadequate, we make use of a procedure based on the variation of elements and seven-fold integration.

Integration of the motion of a comet of the Jupiter family by Cowell's method, with a variable step and considering perturbations by the eight major planets, can be done on the BESM-4 computer at a rate of about $60 \mathrm{yr}$ per hour. For a Trojan minor planet the rate is about $400 \mathrm{yr}$ per hour. Full processing of the observations, including the computation of the solar coordinates, is done at the rate of 14 observations per minute.

The entire set of programmes has been thoroughly checked for proper operation and adequate accuracy. The accumulation of rounding errors has been estimated; it was shown that the rounding error will not build up to the seventh decimal place after 1000 integration steps and will be equal to several units in the sixth decimal place after 10000 steps on the BESM-4 computer.

As already stated, the mean integration step for comets with a perihelion distance of $1.5 \mathrm{AU}$ or more is about 15 days. This amounts to 25 steps per year (or 1000 steps in $40 \mathrm{yr}$ and 10000 steps in $400 \mathrm{yr}$ ). Our programmes may therefore be used for constructing accurate numerical theories for the motion of a short-period comet for some 30 to $40 \mathrm{yr}$ on each side of the initial osculation epoch; i.e., for a total interval of 70 to $80 \mathrm{yr}$, provided that there are no close encounters with Jupiter during that interval. General studies of orbital evolution may be extended over several centuries. In cases where our programmes cannot be utilized (either for the problem as a whole or at particular stages), the double-precision programme by Kazimirchak-Polonskaya should be applied. In order to make a thorough check of the operation of the two programmes, we have integrated the equations of motion of P/Daniel from 1909 to 1944 and compared the integration results; see Table II. There was only a single encounter with Jupiter, to about 0.65 AU in 1912.

The whole range of our programmes has been widely utilized in the studies of the orbital evolution of a large number of asteroids (this work being still in progress); and we have calculated ephemerides, constructed numerical theories, and investigated 
the motions of several comets, notably P/Faye, P/Giacobini-Zinner, P/Daniel, P/Borrelly, P/Tempel-Tuttle and P/Ashbrook-Jackson.

\section{TABLE II}

Comparison of the results of integration of the equations of motion for P/Daniel from 1909 to the epoch 1944 January $31.0 \mathrm{UT}$

\begin{tabular}{lccc}
\hline & $\begin{array}{l}\text { Kazimirchak- } \\
\text { Polonskaya }\end{array}$ & Belyaev & Difference \\
\hline$M_{0}$ & 10.07941 & 10.07874 & $+0^{\prime \prime} 11$ \\
$\omega$ & 6.13719 & 6.13715 & +0.14 \\
$\Omega_{0}$ & 70.43119 & 70.43119 & 0.00 \\
$i$ & 19.84852 & 19.84852 & 0.00 \\
$\nu$ & 35.07173 & 35.07174 & -0.04 \\
$\mu$ & 0.14492861 & 0.14492852 & +0.0003 \\
\hline
\end{tabular}

$e=0.6, q=1.4 \mathrm{AU}, Q=5.6 \mathrm{AU}, P=6.5 \mathrm{yr}$.

\section{Acknowledgments}

It is my pleasant duty to thank E. I. Kazimirchak-Polonskaya and N. A. Bokhan for their continuous interest and help in my work as well as for useful discussions preparatory to the Symposium. My special thanks are also due to N. A. Bokhan, T. I. Podunova and M. Ya. Shmakova, who were kind enough to contribute their standard programmes.

\section{References}

Bokhan, N. A.: 1972, this Symposium, p. 86.

Dubyago, A. D.: 1949, Opredelenie Orbit, Moscow. (English translation: 1961, The Determination of Orbits, Macmillan, New York.)

Kazimirchak-Polonskaya, E. I.: 1967, Trudy Inst. Teor. Astron. 12, 37.

Kulikov, D. K.: 1964, Byull. Inst. Teor. Astron. 7, 782. 